Received: 13 August 2018

Accepted: 21 January 2019

Published online: 27 February 2019

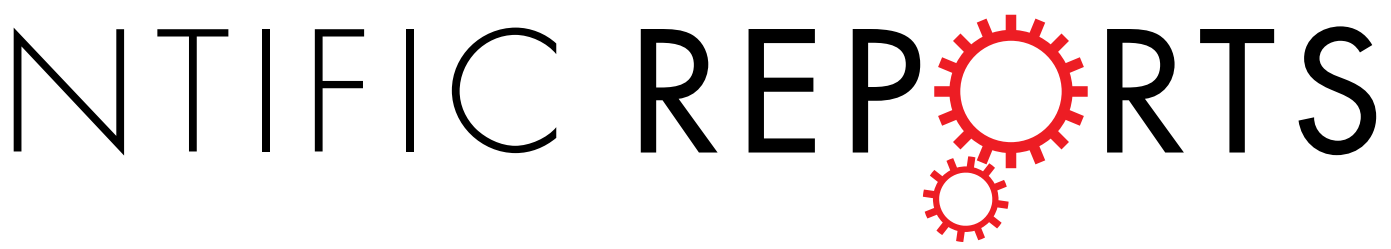

\title{
Polydopamine-based Implantable Multifunctional Nanocarpet for Highly Efficient Photothermal- chemo Therapy
}

Arjun Prasad Tiwari, ${ }^{1,2}$, Deval Prasad Bhattarai ${ }^{3,4}$, Bikendra Maharjan $^{3}$, Sung Won Ko
Hak Yong Kim

We report a design and fabricate multifunctional localized platform for cancer therapy. Multiple stimuli-responsive polydopamine (PDA) was used for surface modification of electrospun doxorubicin hydrochloride (DOX) loaded polycaprolactone (PCL) fibers to make a designated platform. Photothermal properties such as photothermal performance and stability of the resulting composite mats were studied under the irradiation of the near-infrared (NIR) laser of $808 \mathrm{~nm}$. With the incorporation of PDA into the fiber, a remarkable increase of local temperature was recorded under NIR illumination in a concentration-dependent manner with excellent stability. Drug released assay results revealed PDA coated PCL-DOX mats showed pH and NIR dual responsive behavior thereby exhibiting improved drug release in an acidic medium compared to physiological $\mathrm{pH}$ condition ( $\mathrm{pH} 7.4)$ which is further increased by NIR exposure. The cancer activity in vitro of the mats was evaluated using cell counting (CCK) and live and dead cell assays. The combined effect of NIR mediated hyperthermia and chemo release resulting improved cells death has been reported. In summary, this study presents a major step forward towards a therapeutic model to cancer treatment utilizing $\mathrm{pH}$ and NIR dual responsive property from PDA alone in a fibrous mat.

Recently, regional drug delivery (RDD) is gaining much more interest in solid cancer treatment over its conventional drug delivery system. Nanoparticulate drug delivery system as a conventional drug delivery system encounters multiple challenges to complete tumor growth inhibition and eventual tumor eradication owing to rapid clearance, rapid uptake by the reticuloendothelial system and improper drug distribution ${ }^{1,2}$. To this end, RDD has been an iconic platform to modulate the drug on temporal basis matching the physiological needs while reducing the common toxicities associated with chemo drugs to the healthy tissues, thereby enhancing the therapeutic efficacy. Electrospun nanofibers are becoming a device of interest in drug delivery applications because of their versalities of easy fabrication, tunable nano/microstructures, controlled fabrication, improved drug loading, customizable mechanical properties and implantable properties ${ }^{3-8}$. However, controlled and precise drug delivery is a difficult issue from only drug-loaded fibers. For example, hydrophobic PCL or polyurethane (PU) fibers can allow the slow and prolonged drug release for up to months ${ }^{9,10}$. But for the cancer treatment, this cannot be good enough to control the tumor growth due to heterogeneous tumor structure and aggressive growth of tumor cells. The rationally controlled drug release from implantable delivery systems is essential to avoid excessive or deficient drug release and ensure a certain therapeutic drug concentration at the tumor site for an extended time. In these consequences, development of smart drug depot that can provide on-demand localized drug release would be a critical choice.

Cancer tissue offers different microenvironmental conditions compared to adjacent normal cells or tissues in terms of $\mathrm{pH}$, oxygen level, and temperature ${ }^{11}$. Several groups have utilized these unique features to modulate

\footnotetext{
${ }^{1}$ Division of Mechanical Design Engineering, Chonbuk National University, Jeonju, 561-756, Republic of Korea. ${ }^{2}$ Department of BIN Convergence Technology, Chonbuk National University, Jeonju, 561-756, Republic of Korea. ${ }^{3}$ Department of Bionanosystem Engineering, Graduate School, Chonbuk National University, Jeonju, 561756, Republic of Korea. ${ }^{4}$ Department of Chemistry, Amrit Campus, Tribhuvan University, Kathmandu, Nepal. Correspondence and requests for materials should be addressed to C.H.P. (email: biochan@jbnu.ac.kr) or C.S.K. (email: chskim@jbnu.ac.kr)
} 
the drug delivery in a precise manner to the aim of achieving control drug release and enhanced efficacy $\mathrm{y}^{4,5,12,13}$. Stimuli-sensitive drug delivery systems are in the crucial drug administration as they can respond to small signs and changes in the adjacent environment. Wang group demonstrated that a mussel-inspired protein PDA coating can finely tailor the $\mathrm{pH}$-responsive loading kinetics and release of charged molecules ${ }^{13}$. However, the release of drug in $\mathrm{pH}$ dependency only cannot be enough to control the tumor growth because tumor tissues physiological conditions are varied from the surface to core. Sometimes tumor develops the resistivity to chemo. Therefore, a combination of $\mathrm{pH}$-responsive drug release with other therapeutic models such as radiotherapy in $\mathrm{RDD}$, alternating magnetic field (AMF) induced hyperthermia, phototherapy, etc. have been applied to ensure destroying complete tumor cells ${ }^{4,12,14-19}$. Core-shell Cu9S5@mSiO2 nanoparticles, loaded with the DOX, were incorporated into PCL and gelatin nanofibers. When the composite fibers were implanted directly to the tumor site, enhanced orthotopic synergistic therapy was achieved by combined DOX release and photothermal effects from the Cu9S5 nanoparticles under $\lambda=980 \mathrm{~nm}$ laser irradiation ${ }^{5}$. We have also reported recently multifunctional platform of electrospun fibers loaded with anticancer drug and alternative magnetic field sensitive iron oxide for combinational anticancer therapy ${ }^{6}$. Results were appealing, combo model was found showing enhanced tumor cells deaths than to their individual therapeutic model. Among the strategies to introduce hyperthermia, photothermal therapy which utilizes the heat generated from laser irradiation of NIR light-absorbing agents to kill cancer cells, has been widely used and exhibited excellent combo therapeutic effects when applied in combination with chemotherapy. In hyperthermia induction purpose, usually, the inorganic compounds such as copper-based compounds $s^{5}$, iron oxides ${ }^{4,6}$, gold nanorods ${ }^{20,21}$, and lanthanide ${ }^{12}$ are commonly incorporated into polymeric membranes. These inorganic compounds can reside on the body for a longer time due to their non-biodegradation profile which can result in adverse side effects. Moreover, these have limitations for long time using and repetitive uses due to fragmenting or melting ${ }^{22}$. Most importantly, inorganic compounds hardly show a multiple stimulus responsive behaviors. Electrospinning of hyperthermia-induced inorganic particles in a polymeric solution is quite difficult due to their inherent aggregation ${ }^{23}$. Similarly, synthesis of such nanoparticles needs additional hard works. Therefore, it remains an ongoing anticipation to find candidates that are biocompatible organic compounds having multiple functionalities such as $\mathrm{pH}$ and/or NIR-triggered drug release and hyperthermia with excellent stability in a physiological condition that can combine in a drug-loaded fibrous matrix.

PDA is a biodegradable, biocompatible, and organic biopolymer which has been widely used in surface modification of biomedical devices due to its catechol group offering attachment to a wide variety of materials including polymers, metal oxides, and semiconductors ${ }^{24}$. Moreover, it shows multifunctional activities, such as $\mathrm{pH}$ and NIR-responsive behavior that is widely used in the biomedicine to successful triggering drug release and hyperthermia ${ }^{13,25,26}$. Mei group demonstrated PDA modified nanostructures containing anticancer drug can be used as a multifunctional co-delivery system for the targeted chemo, gene, and photothermal therapy against multidrug-resistant cancer ${ }^{14,18,19,27,28}$. NIR irradiation falls in the region of $650-1500 \mathrm{~nm}$ which has been widely used in the biomedical fields including tissue engineering and drug delivery because of safe compared to other light waves ${ }^{29}$. NIR triggered hyperthermia is found more user-friendly and effective to control the disease among other hyperthermia generating mechanism. Therefore, it would be a great effort to fabricate the drug-loaded fibrous meshes with multiple stimuli-responsive PDA coating to achieve a complete therapeutic window in a single platform. DOX was used as a model chemotherapeutic drug since it is broadly used to treat a variety of cancers alone or in combination with other treatment modalities. It works by inhibiting the synthesis of nucleic acids within cancer cells, thereby kills the cells ${ }^{30}$. PCL is taken as a polymeric membrane as a drug carrier since it has been extensively used in biomedical purposes owing to its excellent biocompatibility, tunable mechanical properties and slow biodegradation ${ }^{31-34}$. Although PDA has been widely applied in particulate drug delivery systems and hydrogels for photothermal therapy ${ }^{14-19,24,35-38}$, to the best of our knowledge the potential functional use of PDA in localized drug delivery using nanofibrous membranes has not yet been reported. The present study aims to develop smart multiple stimuli-responsive nanofibers that are capable of both $\mathrm{pH} / \mathrm{NIR}$ dependent drug release in a cancer environment and heat generation under the application of NIR triggered phototherapy for the combined treatments of hyperthermia and chemotherapy.

\section{Experimental Section}

Materials. Poly ( $\varepsilon$-caprolactone) (PCL, Mw $=70,000-90,000)$ was obtained from Sigma-Aldrich (USA). 2,2,2-Trifluoroethanol (TFE) was purchased from Alfa Aesar, USA. DOX.HCl was provided by Sigma, Korea. Dopamine hydrochloride was obtained from Sigma (Korea). Live and the Dead cell assay kit were purchased from Sigma, Korea. All other chemicals used were of analytical grade and were used as received.

Fabrication of PCL-DOX mat. Electrospinning solution (20 g) was prepared by dissolving PCL and DOX (10 wt $\%$ ) in TFE. The weight of DOX was set up $10 \%$ of PCL weight. The electrospinning solution was loaded into a 12-mL syringe. Syringe was then fitted onto syringe pump. During the electrospinning process, the solution was ejected from a syringe at a $1.5 \mathrm{~mL} / \mathrm{h}$ flow rate with an applied voltage of $15 \mathrm{kV}$. The electrospun fibers were harvested on a polyethylene sheet that attached on the collector $15 \mathrm{~cm}$ away from the tip of the needle. Electrospinning was carried out in the chamber under stable conditions of temperature $\left(\mathrm{T}=25 \pm 1.5^{\circ} \mathrm{C}\right)$, and relative humidity $(30 \pm 5 \%)$. Then as-prepared electrospun membranes were dried in a vacuum oven at $35^{\circ} \mathrm{C}$ for $24 \mathrm{~h}$. The mats obtained from electrospinning are referred to as PCL-DOX mat (PDP0). Similarly, pure PCL mat was also prepared under the same preparation conditions.

Surface Modification of Fiber by PDA. Surface modification of the PCL-DOX mat (PDP0) by PDA is presented in Fig. 1. The desired size of the PDP0 mat was placed in a petridish containing $50 \mathrm{~mL}$ Tris buffer with different concentration $(4,8$ and $12 \mathrm{mg} / \mathrm{mL}$ in $100 \mathrm{mM}$ Tris. $\mathrm{HCl})$ of dopamine monomer and allowed for $8 \mathrm{~h}$ at $4^{\circ} \mathrm{C}$ for coating. Later, coated mats were washed thoroughly with deionized water and dried in a vacuum oven at 

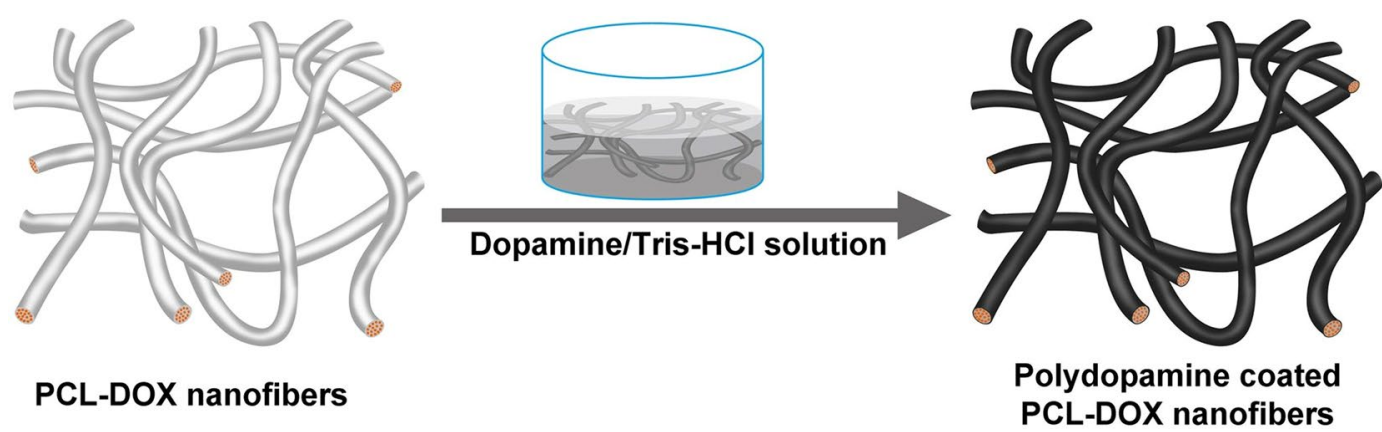

Figure 1. Schematic representation of the fabrication procedure of the PDA modified PCL-DOX nanofibrous mat.

$35^{\circ} \mathrm{C}$ for $24 \mathrm{~h}$. PDA decorated PCL-DOX nanofibrous mats obtained from 4,8 and $12 \mathrm{mg} / \mathrm{mL}$ dopamine in tris buffer are referred to as PDP1, PDP2, and PDP3, respectively. Similarly, PDA coating was performed over pure PCL mat following the same condition as PDP2 fabricated and referred to as PP2 mat.

Physicochemical Properties Study. Field emission scanning electron microscopy (FESEM, Hitachi SU-70, Japan) was used to investigate the structure and morphology of the as-spun PDA coated membranes. The average fiber diameter values from the SEM images were obtained from analyzing by Image J (NIH, USA) software. At least 100 nanofibers in different SEM images for each sample were analyzed to obtain the average fiber diameter. FT-IR experiments were performed at $25^{\circ} \mathrm{C}$ on a PerkinElmer $580 \mathrm{~B}$ infrared spectrophotometry over the spectral region of $400 \mathrm{~cm}^{-1}$ to $4,000 \mathrm{~cm}^{-1}$. Crystalline properties of the membranes were evaluated by an X-ray diffraction instrument (XRD, Rigaku X-ray diffractometer). Elemental identification of the PDA coated PCL-DOX mat was analyzed using an Axis Ultra DLD X-ray photoelectron spectrometer (XPS; Kratos, U.K.) equipped with monochromatic Al-K $\alpha$ X-ray excitation source. Thermal gravimetric analysis (TGA, Q50 TA Instruments) was used to investigate the thermal stability of different mats; the samples were heated from 30 to $600^{\circ} \mathrm{C}$ at a rate of $10^{\circ} \mathrm{C} / \mathrm{min}$ under a nitrogen purge of $40 \mathrm{~mL} \mathrm{~min}^{-1}$. Thermal analyses were performed using differential scanning calorimeter (DSC) (TMK 0017 universal analysis TA Instruments Co., USA). 8 mg weight of the sample was always used in DSC analyses. All DSC thermograms were taken under the nitrogen atmosphere. DSC scans were taken from -80 to $300^{\circ} \mathrm{C}$ under nitrogen flow $(50 \mathrm{~mL} / \mathrm{min})$ in all runs with scan rate at $10^{\circ} \mathrm{C} /$ min (first scan). After the samples being quenched at $-80^{\circ} \mathrm{C}$, second scans were made by exposing the samples upto $300^{\circ} \mathrm{C}$. To measure the mechanical properties of the mats, universal testing machine (AG-5000G, Shimadzu, Japan) with a $10 \mathrm{~N}$ load cell was used. For mechanical testing, mats were cut into dog-bone-shaped templates. The template was $40 \mathrm{~mm}$ length and $10 \mathrm{~mm}$ width. A cross-head speed of $5 \mathrm{~mm} / \mathrm{min}$ was used for all tests. Thickness of mats was measured by digital caliper. Each sample was run to the point of failure and the ultimate tensile strength elongation at break and Young's modulus values were obtained automatically.

NIR-Responsive Photothermal Property Study. To study the photothermal effect of different mats, equal weight of samples placed on the $2 \mathrm{~mL}$ of PBS solutions were irradiated by a NIR laser $\left(808 \mathrm{~nm}, 1.5 \mathrm{~W} / \mathrm{cm}^{2}\right)$ for $5 \mathrm{~min}$ with various power doses $\left(1,1.5\right.$ and $\left.2 \mathrm{~W} / \mathrm{cm}^{2}\right)$, separately. The changes of the temperature on the mat was monitored in a real time by an infrared camera (FLIR C-Series). To investigate the photothermal stability of the membranes, two approaches were applied. Firstly, the temperature changes of the mats following ten heating $\left(5 \mathrm{~min}\right.$ at $\left.1.5 \mathrm{~W} / \mathrm{cm}^{2}\right)$ and cooling cycles $(5 \mathrm{~min})$ were monitored. In another way, photothermal stability was studied by comparing the VIS-NIR spectra that linked to stability of the membranes. For that, samples were placed into chloroform for $4 \mathrm{~h}$ and subsequently incubated at $80^{\circ} \mathrm{C}$ to remove PCL-DOX part. Thus, obtained PDA coating was washed, dried and minced to get $100 \mu \mathrm{g} / \mathrm{mL}$ suspension which was subjected to VIS-NIR analysis.

Drug Release Study. Different mats each of $2 \mathrm{~cm}^{2}$ size were placed on the $15 \mathrm{~mL}$ tube containing PBS solutions with different $\mathrm{pH} 5.5,6.8$ and 7.4 and incubated for different time intervals at $37^{\circ} \mathrm{C}$ in a shaking condition $(120 \mathrm{rpm})$. At designated time points, whole release medium was collected and replaced with an equal volume of fresh PBS solution. UV-VIS spectrophotometry was used for measurement of the DOX concentration in the collected medium. NIR mediated release was assayed by applying the NIR into the mats that had been kept for different $\mathrm{pH}$ of PBS. Drug release experiments were performed three times. Obtained data were plotted as a cumulated release versus laser power (NIR) and time, separately.

Cell Culture. Human lung cancer cells (A549) and a human breast cancer cell line (MCF7), were maintained as a monolayer culture in DMEM medium supplemented with $10 \%$ fetal bovine serum (Gibco) and $1 \%$ penicillin-streptomycin (Gibco) at $37^{\circ} \mathrm{C}$ in a humidified atmosphere $\left(5 \% \mathrm{CO}_{2}\right)$.

In Vitro Localized Photothermal Cytotoxicity. The localized photothermal cytotoxicity of the PDA coated mats was evaluated using A549 and MCF7 cells with and without NIR laser illumination. Later, live/dead cell viability and CCK-8 assays were performed to monitor cytotoxicity according to our previous reports s $^{7,39}$. Membrane with $1 \mathrm{~cm}^{2}$ in size was attached to round coverslip, and placed into 48-well plate. Samples were ensured to sterilize by UV exposure for $2 \mathrm{~h}$ and subsequent washing with sterile PBS. Then, cells were seeded $(50,000$ cells/well) separately in the wells of a 48 -well plates containing mats and allowed to incubate for $24 \mathrm{~h}$. Cells were 

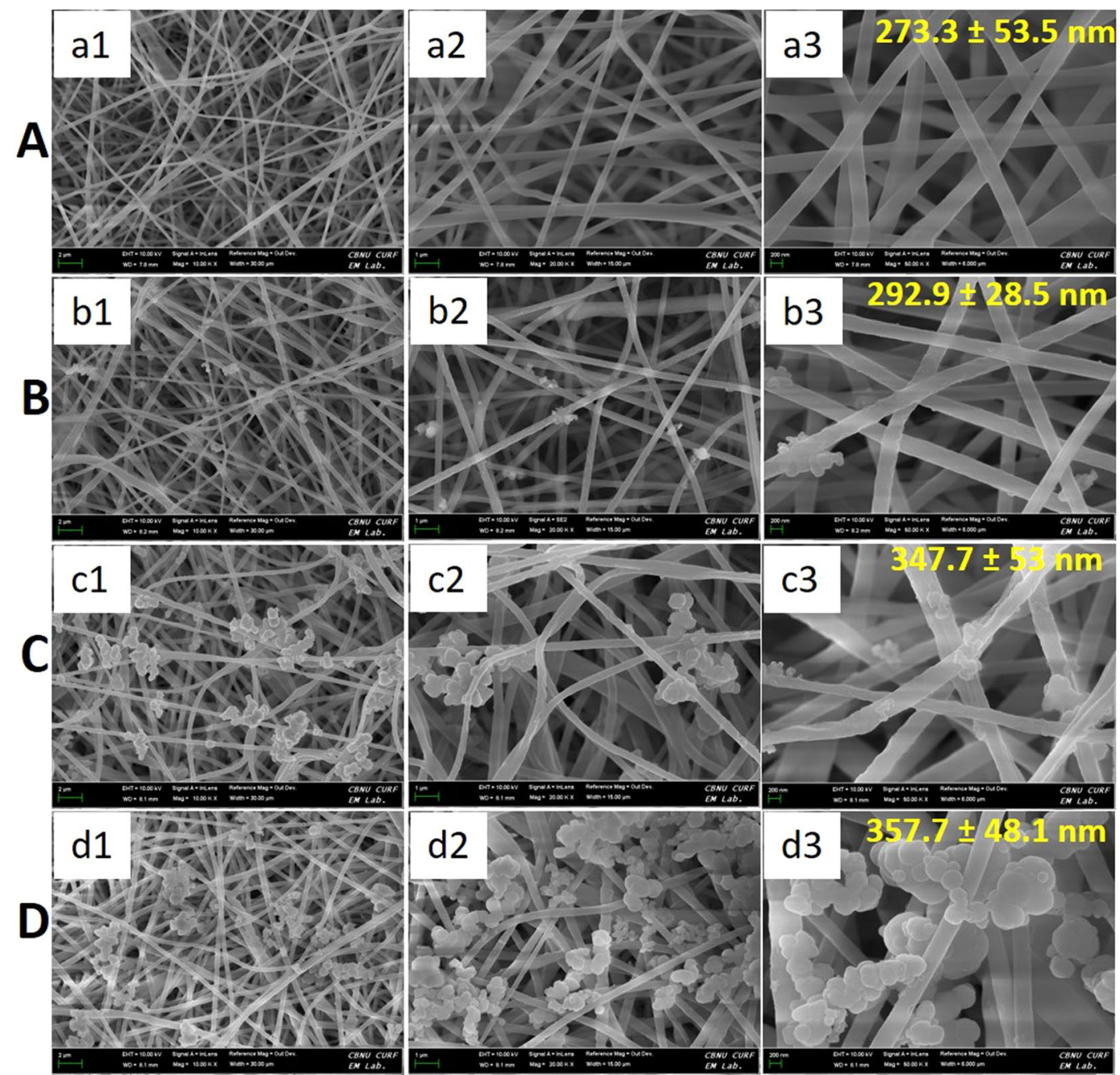

Figure 2. FESEM images of different mats at different magnifications. PDP0 (panel A), PDP1 (panel B), PDP2 (panel C) and PDP3 (panel D).

irradiated with NIR laser of varied intensities $\left(1,1.5\right.$ and $\left.2 \mathrm{~W} / \mathrm{cm}^{2}\right)$ separately for $5 \mathrm{~min}$. Cell viability was quantitatively measured another $24 \mathrm{~h}$ of photothermal treatment, using a CCK- 8 assay according to the manufacturer's instructions. Each experiment was performed in triplicate, and the data represent the mean \pm standard error $(\mathrm{n}=3)$. The untreated corresponding cells grown in tissue culture plate (48 well) were taken as control with $100 \%$ viability. We have further evaluated the IC50 values of different fibrous mats for MCF7 cells in designated condition. The drug concentration at which the growth of 50\% cells was inhibited (IC50) in comparison with that of the control sample was calculated by curve fitting of the cell viability data ${ }^{19,40}$. Similarly, live and dead cell assay was performed to support the CCK-8 data. For the live and dead assay, MCF7 cells were washed three times with PBS, followed by staining with resazurin and SYTOX green according to the manufacturer's protocol. Stained samples were observed by confocal microscopy.

Statistical Analysis. All data are presented as a mean \pm standard error $(n=3)$ unless otherwise mentioned. One-way analysis of variance (ANOVA) was used to analyze the significance of the difference between groups. P value less than 0.05 is considered statistically significant.

\section{Results and Discussion}

Fabrication and Characterization of PDA Coated Mats. In this work, we chose the PCL as a model polymer to make an electrospun fiber, a drug carrier, due to its abundant uses in biomedical applications including drug delivery. PCL is a biocompatible, biodegradable, and non-immunogenic polymer with excellent mechanical properties ${ }^{31,41,42}$. Electrospun fibrous meshes oversee to use as a drug depot due to their high loading efficiency and allowing controlled and prolonged drug release. Firstly, PCL and DOX were blended and electrospun. Resulting PCL-DOX fibers are uniform in diameters without any secondary structures as confirmed by FESEM (Fig. 2A). The average fiber diameter is found to be $273.3 \pm 53.5 \mathrm{~nm}$. 

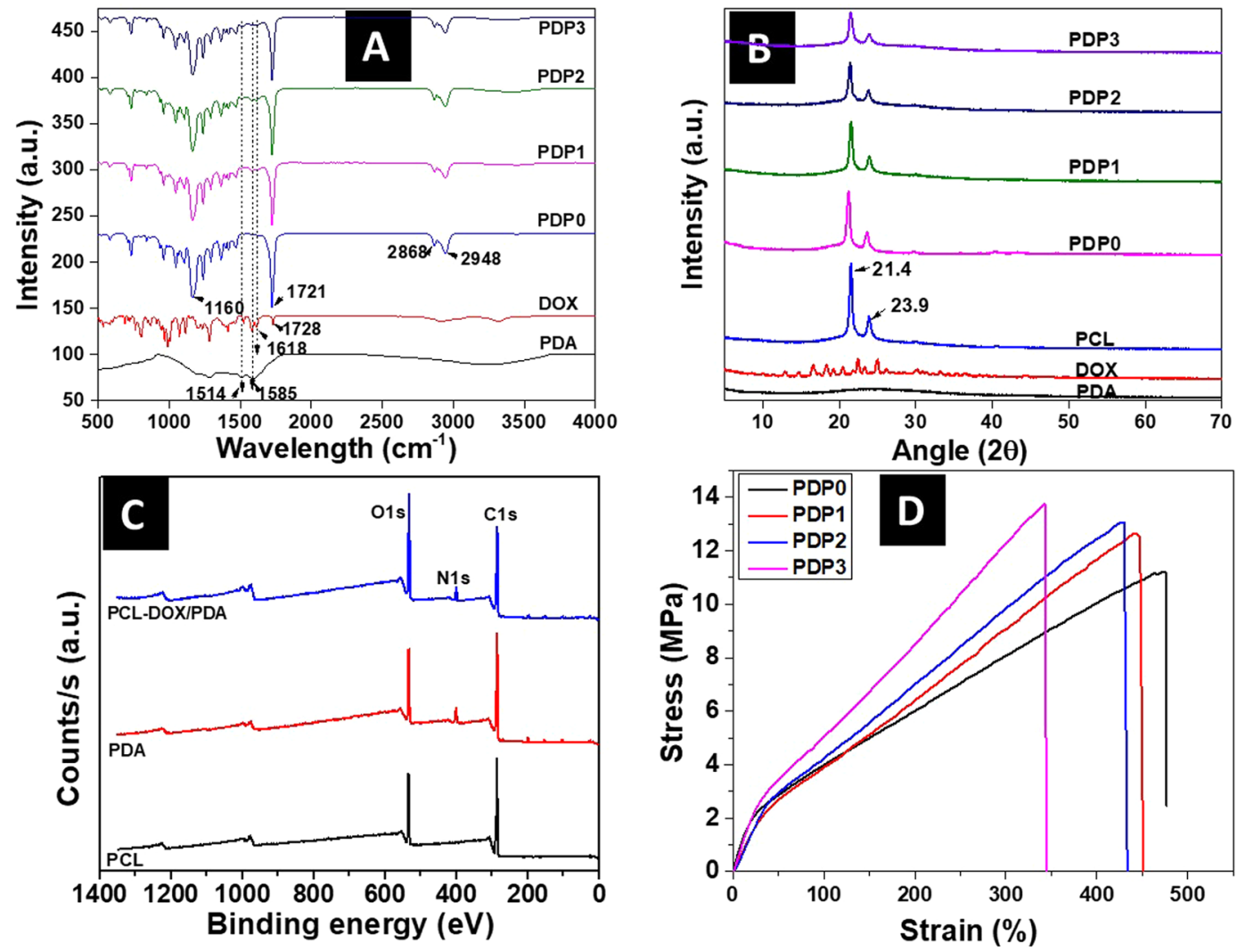

Figure 3. (A) FT-IR spectra, (B) XRD patterns, (C) XPS spectra and (D) Stress-strain curves of different samples.

To grab the abundant possibilities of PDA coating in order to make a multifunctional platform, PDP0 fibers were coated with various concentration of PDA by the chemical polymerization of dopamine. The PDA coated PDP0 meshes obtained from the polymerization of dopamine of 4,8 , and $12 \mathrm{mg} / \mathrm{mL}$ concentration are named as PDP1, PDP2, and PDP3, respectively. Figure 2 shows the influence of the concentration of dopamine on the surface morphology of the fibers. Each of the PDA coated meshes exhibited consistent fiber diameters (Fig. 2(B-D)). However, Fiber diameters are observed gradual increased with the increased concentration of dopamine. Average fiber diameter was found increased from $273.3 \pm 53.5 \mathrm{~nm}$ of PDP0 to $292.9 \pm 28.5,347.7 \pm 53.0,357.7 \pm 48.1 \mathrm{~nm}$ for PDP1, PDP2 and PDP3, respectively. In addition of a thin coating of PDA layer, some nodules like PDA particle- aggregates appear higher extent in PDP3 (Fig. 2D) compared to PDP2 (Fig. 2C) and PDP1 (Fig. 2B). The successful entrapment of DOX and coating of the PDA on PCL fibers was verified by FT-IR measurement (Fig. 3A). A new peak assigned to the DOX appears at the $1618 \mathrm{~cm}^{-1}(\mathrm{C}=\mathrm{C} \text { band })^{43}$ in composites, confirms the loading of DOX into PCL fibers. Moreover, $1728 \mathrm{~cm}^{-1}(\mathrm{C}=\mathrm{O} \text { band })^{43}$ for DOX is shifted to lower band at $1721 \mathrm{~cm}^{-1}$, can be related to the adsorption of DOX on the PCL. Moreover, most of the peaks associated with DOX are becoming reduced or disappeared possibly due to overlapping of the carrier polymer PCL or coating agent, PDA (Fig. 3A). Similarly, after modification of the PDA layer on the fiber surface, additional peaks assigned to PDA appears at $1585 \mathrm{~cm}^{-1}(\mathrm{C}=\mathrm{C} \text { stretching mode })^{44}$, confirming the coating of a PDA layer on PDP0 fibers. Absorption bands at 2948 and $2868 \mathrm{~cm}^{-1}$ (stretching bands of $\mathrm{CH}_{2}$ groups), $1721 \mathrm{~cm}^{-1}$ (stretching band of the ester group $\mathrm{C}=\mathrm{O}$ ), and $1163 \mathrm{~cm}^{-1}$ (asymmetric and symmetric stretching of $\mathrm{C}-\mathrm{O}-\mathrm{C}$ ) are associated with the PCL in PDP0 sample ${ }^{31,45}$. An apparent reduction in the intensities of all peaks corresponding to the PDP0 matrix were observed in a concentration-dependent manner once coating with PDA (Fig. 3A). Figure 3B shows the XRD spectra of different samples. PDA did not show any crystalline peak, suggests it is amorphous in nature. PCL exhibited the typical XRD peaks at 21.4 and $23.9^{\circ}$ (Fig. 3B) revealing its crystalline nature, consistent with other reports $^{46}$. Loading of DOX into the PCL fibers and/or subsequent modification of PCL fiber by PDA coating has not changed the peaks position, suggesting there was almost no change to the crystalline region in the PCL chain. Pure DOX shows its crystalline nature, however, typical peaks of DOX were disappeared in PDP0 fibers. This may be due to the entrapment of the DOX into the matrix to a much lesser extent compared to PCL which is consistent to others works ${ }^{8,47}$.

XPS was used to confirm the surface modification of PCL-DOX by PDA coating. The XPS spectra of pure PCL, pure PDA, and PCL-DOX/PDA are shown in Fig. 3C and Table 1. XPS spectra of PCL-DOX/PDA reveal the presence of all spectra corresponding to PCL (O1s and C1s peak BE at 533.95 and $286.27 \mathrm{eV}$, respectively) and PDA (O1s, N1s and C1s peak BE at 533.37, 401.03 and $286.12 \mathrm{eV}$ respectively). It is worth noting that the elemental analysis of composite mat reveals the increase of the atomic percentage of oxygen and decrease of nitrogen as 


\begin{tabular}{|c|c|c|c|c|c|c|}
\hline \multirow[b]{3}{*}{ Materials } & \multicolumn{6}{|c|}{ Elements with their peak binding energy (BE) and atomic percentages. } \\
\hline & \multicolumn{2}{|l|}{ O1s } & \multicolumn{2}{|l|}{ N1s } & \multicolumn{2}{|l|}{ C1s } \\
\hline & Peak BE (eV) & At $\%$ & Peak BE (eV) & At $\%$ & Peak BE (eV) & At $\%$ \\
\hline PCL & 533.95 & 21.14 & - & - & 286.27 & 78.08 \\
\hline PDA & 533.37 & 19.09 & 401.03 & 7.24 & 286.12 & 69.99 \\
\hline PCL-DOX/PDA & 532.70 & 26.52 & 400.40 & 5.98 & 285.85 & 65.02 \\
\hline
\end{tabular}

Table 1. XPS spectra of different samples.

\begin{tabular}{|l|l|l|l|}
\hline Materials & $\begin{array}{l}\text { Elongation at } \\
\text { break (\%) }\end{array}$ & $\begin{array}{l}\text { Young's modulus } \\
\text { (MPa) }\end{array}$ & $\begin{array}{l}\text { Tensile } \\
\text { strength (MPa) }\end{array}$ \\
\hline PDP0 & $477.1 \pm 27.1$ & $10.4 \pm 1.9$ & $11.2 \pm 1.9$ \\
\hline PDP1 & $450.2 \pm 29.3$ & $7.3 \pm 0.8$ & $12.6 \pm 0.9$ \\
\hline PDP2 & $434.3 \pm 28.5$ & $6.4 \pm 1.3$ & $13.0 \pm 1.5$ \\
\hline PDP3 & $343.3 \pm 37.4$ & $10.1 \pm 1.3$ & $13.6 \pm 1.3$ \\
\hline
\end{tabular}

Table 2. Mechanical properties of the samples. Note: Each sample was evaluated in triplicate. The mean value and corresponding standard deviation are presented.
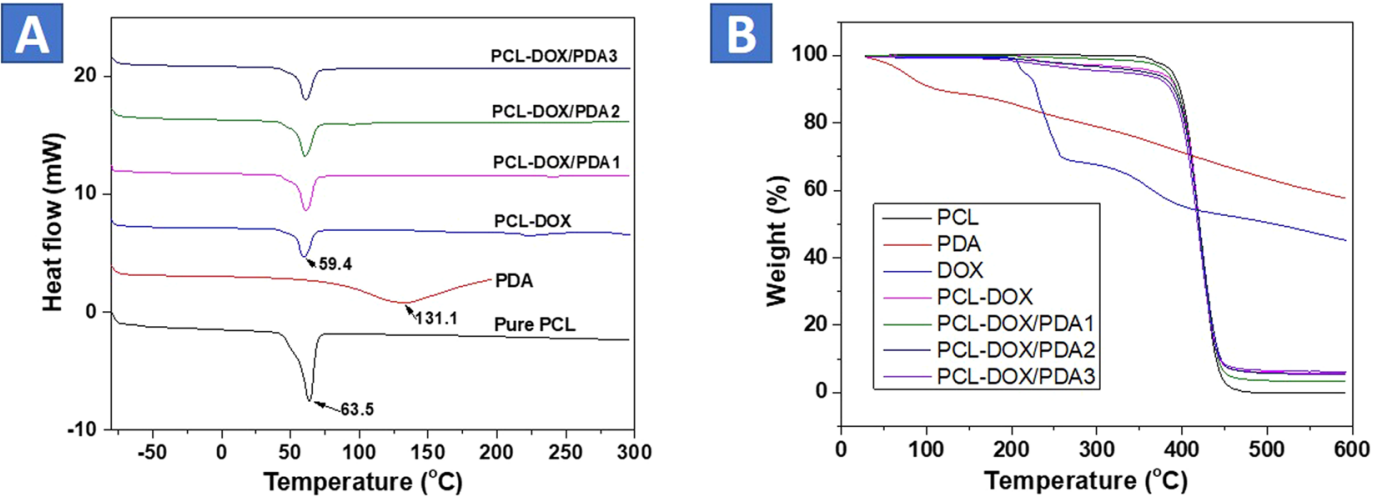

Figure 4. Thermal behavior of samples. (A) DSC and (B) TGA curves.

compared to PDA. Meantime, a decrease of carbon content was noticed for composite mat as compared to PCL and PDA (Table 1). These results are due to the coating of PDA layer over the PCL-DOX mat ${ }^{15,16,48,49}$.

Mechanical Properties Study. Mechanical property plays a key role behind the success of any localized therapeutic platform, as forces exerted on the fibers may result in permanent deformation or even failure during the service time of the fibers. Herein, mechanical properties were measured using the mechanical tensile tester. The stress-strain curves of the different fibrous membranes are shown in Fig. 3D. Table 2 presents the mechanical properties including Young's modulus, tensile strength, and elongation at breaks (\%). Data reveal the tensile strength of PDP0 is increased from 11.2 MPa gradually to 12.6, 13 and 13.6 MPa for the PDP1, PDP2 and PDP3, respectively (Fig. 3D and Table 2), indicating stronger materials formed after modification with PDA. However, the strain of PDP0 mat was observed in opposite trend of stress when coated with PDA (Fig. 3D). The attachment of the PDA as a film reduced the mobility of the polymeric chains by improving interconnection among the fibers, thereby reduced the elasticity of PDP0 fiber ${ }^{50}$. However, same property of PDA may result in the improved tensile properties indicated by toughness in PDA modified fibers. PDP3 exhibited the higher Young's modulus compared to the PDP0 mat (Table 2), suggests the increased stiffness than that of PCL. However, PDP1 and PDP2 have shown lesser Young's modulus than the PDP0 mat. Results in the average revealed deposition of the PDA on to the drug-loaded mat can show the reinforcement effect. Tensile strength more than $10 \mathrm{MPa}$ and Young's modulus more than 6.5 MPa with appropriate elasticity can be good enough to support the soft and cartilages types of tissues ${ }^{51}$.

Thermal Behavior Study. Thermal behavior of the samples was studied by DSC and TGA to understand the thermal behavior (Fig. 4). Figure 4A represents a DSC data. DSC data of PCL showed the endothermic peak at around $63^{\circ} \mathrm{C}$, is attributed to the semicrystalline PCL. With the incorporation of DOX into PCL fibers, slightly blue shifting of the peak $\left(59.4^{\circ} \mathrm{C}\right)$ occurred, is associated with the decreasing crystallinity of PCL fibers. The PCL and DOX are incompatible in solubility issue, therefore, the heterogeneous distribution of the DOX molecules into PCL matrix could result in the reduced crystallinity of PDP0 fiber ${ }^{52}$. A broad peak is observed for PDA as shown in Fig. 4A, represents its amorphous form. However, Further grafting of the PDA layer an endothermic 

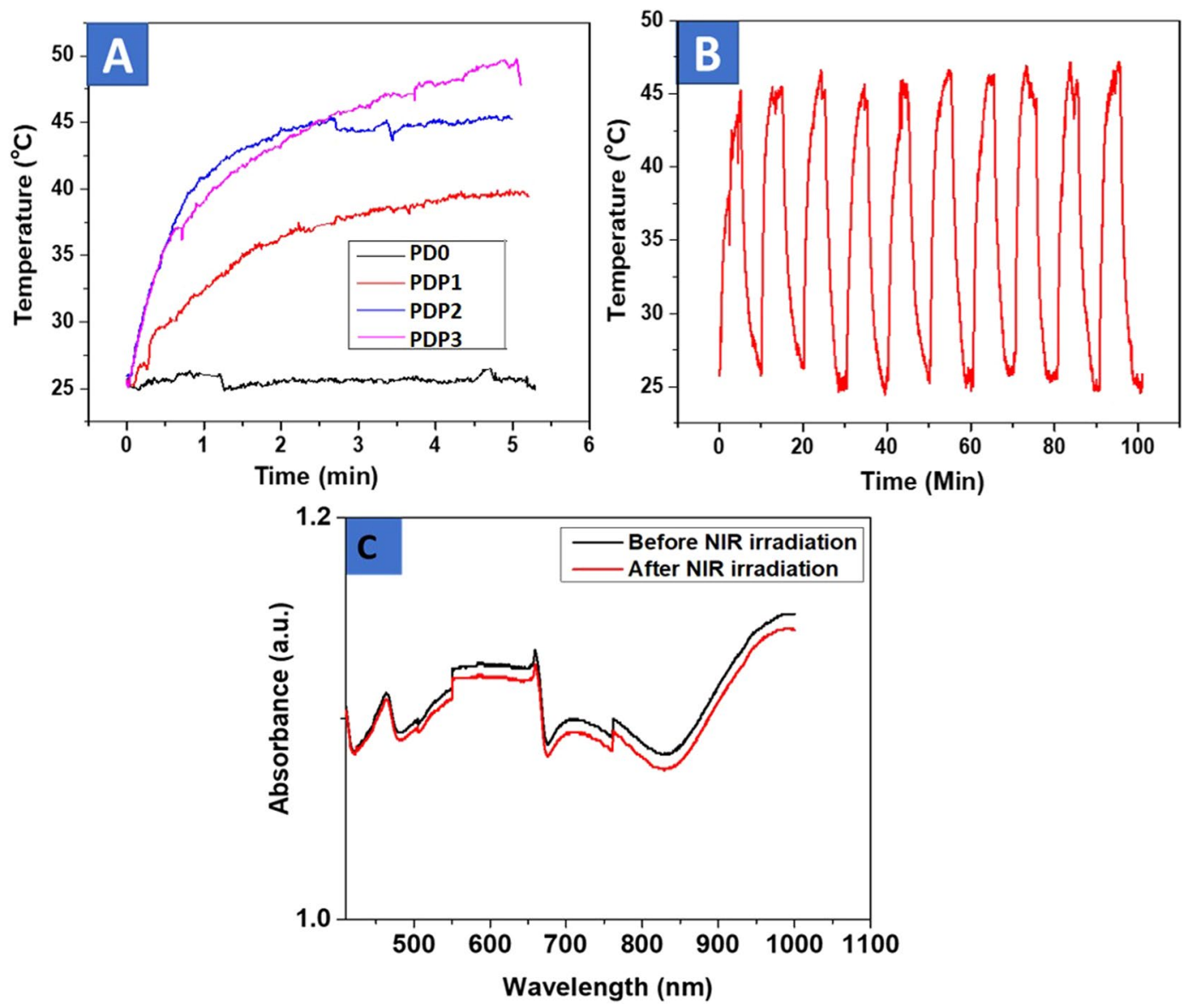

Figure 5. NIR-responsive Property Tests (A) Heating curves of different PDP2 mats, (B) Stability test; changes of the temperature profiles of PDP2 mat (PDP2) as a function of NIR on-off cycles (NIR on time: 5 min; NIR off time: $5 \mathrm{~min}$ ) and (C) UV-VIS-NIR spectra of PDP2 before and after cycles of NIR irradiation. In this experiment, NIR laser of $808 \mathrm{~nm}$ wavelength with $1.5 \mathrm{~W} / \mathrm{cm}^{2}$ power density was irradiated.

peak $\left(59.4^{\circ} \mathrm{C}\right)$ of PDP0 matrix was shifted to slightly higher temperature $\left(61.2^{\circ} \mathrm{C}\right)$, can be explained by slightly increasing crystallinity. The formation of transcrystalline features occurred in interfaces of PDA and PDP0 fibers can improve the crystallinity of the polymer ${ }^{53}$. However, the concentration of PDA coating was observed independently to changes of crystallinity. Figure $4 \mathrm{~B}$ shows the TGA curves of different mats obtained after surface modification of PDP0 fibers. The figure shows only one major weight-loss stage was observed for pure PCL starting decomposition from $356^{\circ} \mathrm{C}$ to complete annihilation at $427^{\circ} \mathrm{C}$. All samples lost some weight below $200^{\circ} \mathrm{C}$, is attributed to the moisture and solvent evaporation. Pure DOX showed four phase degradations. Only $55 \%$ was observed degradation heating up to $600^{\circ} \mathrm{C}$.

The Addition of the DOX into PCL exhibited obviously increased degradation temperature (Fig. 4B). Similarly, PDA shows 3 transitions for degradation, first degradation about $13 \%$ mass loss was occurred up to $179^{\circ} \mathrm{C}$, another $8 \%$ was degraded heating up to $324^{\circ} \mathrm{C}$ and another major loss about $22 \%$ was observed after $324^{\circ} \mathrm{C}$. The significant mass loss in the last transition is associated with the degradation of the main PDA chain ${ }^{54}$. It is worth noting that mass residue was high as $57 \%$. The PDP2mats exhibited decreased weight loss (residue $5 \%$ ) at the temperature range compared to zero residues for the PCL fibers, indicating the loading of DOX and deposition of PDA layer could result in increased thermostability of PCL fibers. Because of the high thermal stability of PDA, the PDA film can protect the PCL-DOX fiber from being degraded, thus increases the degrading temperature.

NIR-Responsive Photothermal Property Study. Since our motive is to evaluate the photothermal performance of the resulting composite membranes, it is important to understand their photothermal conversion capability and stability under the NIR irradiation. Photothermal properties were monitored based on the temperature elevation under NIR irradiation by FLIR camera. The temperature of composite mats PDP1, PDP2 and PDP3 were found increases by $13.7,20.4$ and $24.9^{\circ} \mathrm{C}$, respectively, after exposure to a NIR laser of $1.5 \mathrm{~W} / \mathrm{cm}^{2}$ for 5 min while no apparent increases of the temperature of the PDP0 mat was recorded i.e. stays between $25-26^{\circ} \mathrm{C}$ (Fig. 5A). Moreover, the gradual increases of the temperature under NIR were observed with increasing time of exposure (Fig. 5A). These data indicate the PDA coating of drug-loaded fibers increases the photothermal conversion capability in the PDA concentration and time-dependent manner. The photothermal conversion ability of the PDA coated fibers is related to the strong NIR absorbing capability of PDA, which has been reported by many groups ${ }^{25,26}$. Based on the temperature profile fibers had shown, PDP2 was selected for proceeding experiments 

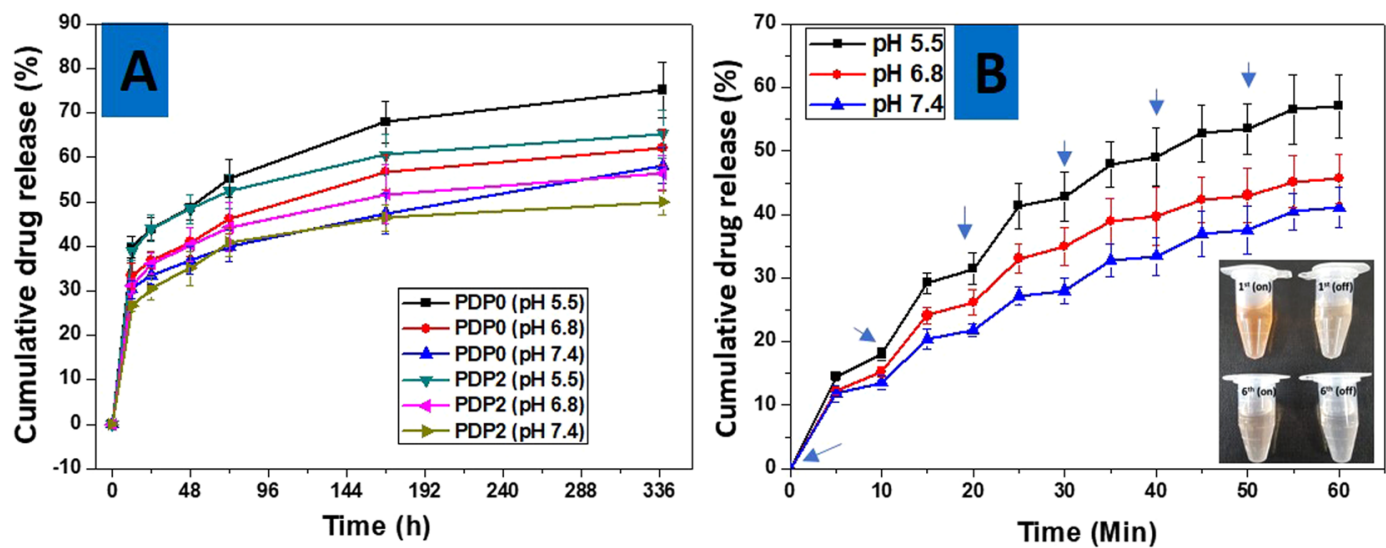

Figure 6. Drug release behavior. (A) DOX release profile from PCL-DOX (PDP0) and PCL-DOX/PDA (PDP2) membranes in PBS solutions at different $\mathrm{pH}$ values, and (B) DOX release profiles from PDP2 membranes as a function of NIR on-off cycles. Arrows show the irradiated time points. Digital images in Figure B (inset) are the representative released solution in first and six cycles when conducted at pH 5.5 PBS solution. Here, Data are expressed as mean values \pm standard error $(n=3)$.

because for a hyperthermia treatment temperature range of $42-46{ }^{\circ} \mathrm{C}$ is reported as an appropriate. In this temperature ranges, cells are undergone to apoptosis while higher than that ranges leading cell deaths via necrosis ${ }^{55}$. Since inadequacy to kill the tumor cells owing to their aggressive nature and chemo resistance of tumor cells, repeated treatment is needed, herein, the evaluation of photothermal stability is a crucial parameter for therapy. The mat was undergone on-off of NIR irradiation for 10 cycles ( $5 \mathrm{~min}$ on followed by $5 \mathrm{~min}$ off) and temperature elevation was checked each time (Fig. 5B). Results showed maximum temperature level remained consistent about $46^{\circ} \mathrm{C}$ in each exposure time while it shows temperature fall down consistently under NIR off mode, indicating excellent stability of PDA coated PCL/DOX fiber under NIR. Furthermore, stability of the membranes was studied by UV-VIS-NIR spectroscopy. VIS-NIR spectra were taken before and after the membranes undergone of stability test (Fig. 5C).

Figure 5C shows VIS-NIR spectra of membranes, where a strong absorption in the near infrared wavelength region can be found at $800 \mathrm{~nm}$ beyond. NIR related peak position and intensity were observed remained consistent with each other, indicates stability of the mats under NIR exposure. The heating reproducibility and high photothermal stability of as-synthesized PDA modified PCL-DOX mats demonstrate the great potential as photothermal agents.

Drug Release Study. DOX was chosen as a model drug for investigating the drug release ability of electrospun PCL membrane coated with PDA since it has a broad-spectrum anticancer activity used in the treatment of a variety of solid tumors. The encapsulation efficiency of the DOX was obtained $90 \%$ and $~ 75 \%$ for PDP0 and PDP2 fibers. Figure 6A shows the cumulative drug release (\%) from PDP0 and PDP2fibers into three different release media having different $\mathrm{pH}$ values; $\mathrm{pH}=5.5,6.8$ and 7.4. Both fibers showed the drug released in a biphasic profile with initial burst release, which continued for $24 \mathrm{~h}(30-40 \%)$, the following time interval showed a slow and prolonged release at all $\mathrm{pH}$ conditions. Burst drug releases from the fibers are attributed to the quickly shedding of the drug that remained on the surface of the fiber. The results exhibited there was increased release was observed in PCL-DOX than PDP2in all time intervals in all pH conditions. For example, PDP0 showed 68.0, 56.7, 47.3\% cumulative release in $\mathrm{pH}$ 5.5, 6.8 and 7.4 during 1-week incubation, respectively. However, DOX released was substantially decreased with PDA coating, reached 60.5, 51.7 and 46.4\% in pH 5.5, 6.8 and 7.4, respectively in the same period (Fig. 6A). This is obvious, PDA coating prevents the drug release from initially because it acts as a barrier between medium and drug. Moreover, a released drug from the PCL-DOX fiber can be adsorbed by the PDA layer. The aromatic compound can interact with the conjugated polymer via $\pi-\pi$ interaction ${ }^{56}$. Results further indicate the solution $\mathrm{pH}$ has influenced the release kinetics, the release was observed faster in a low $\mathrm{pH}$ in both PDP0 and PDP2 fibers. The solubility of DOX is improved with decreasing $\mathrm{pH}$ of the solution due to protonation of $-\mathrm{NH}_{2}$ groups on the $\mathrm{DOX}^{57}$, thereby increasing release was obviously observed higher in low $\mathrm{pH}$. DOX release was continued 2 weeks period reaching the highest $75 \%$ to PDP0 at pH 5.5 which is 13 and $17 \%$ higher than its counterpart fiber that has been tested at pH 6.8 and 7.4, respectively. In the same period (2 weeks) PDP2 in pH 7.4 showed the lowest value which is 16 and 7\% lower than its counterpart mat that had conducted at pH 5.5 and 6.8, respectively (Fig. 6A). These results indicate clearly the drug release from both fibers coated and uncoated is dependent to the $\mathrm{pH}$ of the PBS solution. Increased drug release observed in PDA coated fibers in low $\mathrm{pH}$ solution compared to higher $\mathrm{pH}$ solution can be explained two reasons. First reason can be explained by $\mathrm{pH}$-responsive nature of PDA. At low $\mathrm{pH}$, the amine groups of PDA became protonated and the neutralization of the charges led to an absence or decrease in the ionic interactions with positively charged DOX which resulting in the abrupt release of DOX from the PDA coated fibers ${ }^{15,16,58}$. Another reason could be due to increased solubility of the DOX into the medium at low $\mathrm{pH}$ condition compared to high $\mathrm{pH}$ solution. Given the mild acidic extracellular microenvironment compared with normal tissue, this stimulus-responsive release tendency could provide a beneficial platform in $\mathrm{pH}$-mediated anticancer chemotherapy. 

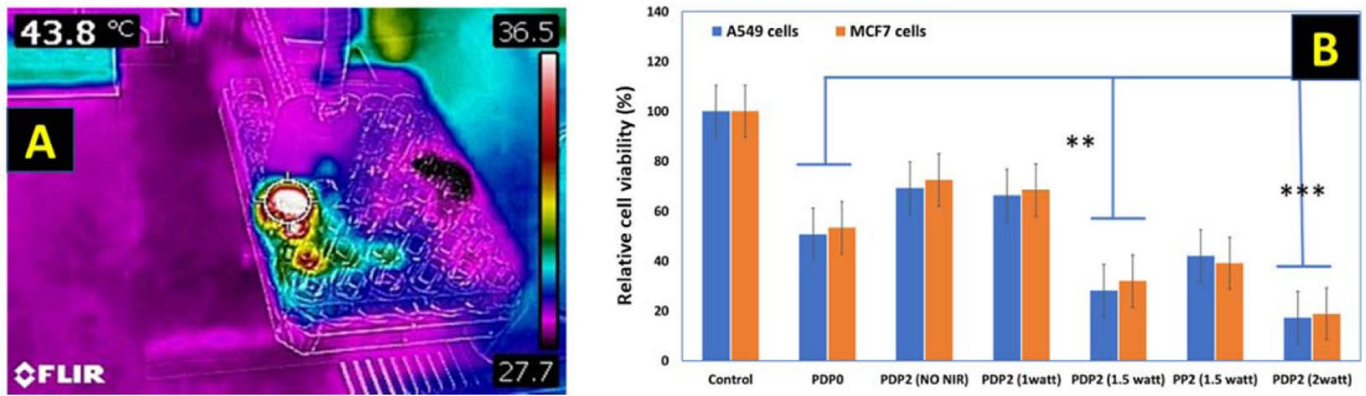

Figure 7. (A) Thermographic image of the cell treatment by NIR device, and (B) Cytotoxic effect of the samples against A549 and MCF7 cells determined by CCK- 8 assay. Data are presented as mean values \pm standard deviation $(\mathrm{n}=3)$.

NIR-Responsive Drug Release Study. The effect of NIR on the PDA coated fibers on PBS of different $\mathrm{pH}$ conditions was studied. PDP0 has not shown any heating property, therefore, these fibers were excluded from NIR illumination. NIR laser of $808 \mathrm{~nm}$ was subjected to PDA coated mats for $5 \mathrm{~min}$ and allowed to subsequent cooling at next $5 \mathrm{~min}$ at room temperature, this cycle was repeated for 6 times. In each point, samples were checked for drug content via UV-VIS spectroscopy. As shown in Fig. 6B, PDA coated mats in all pH conditions exhibited DOX release in on-off basis, with burst release during NIR on-process followed by a negligible release in absence of NIR (NIR off). The drug release was observed continuously decreased in the following cycle (Fig. 6B). The solution $\mathrm{pH}$-dependent release was observed according to Fig. 6B. PDA coated fibers displayed 57.1, 45.6 and $41.08 \%$ release in $\mathrm{pH}$ 5.5, 6.8 and 7.4, respectively. Digital images of the drug released in different cycles shown clearly support the NIR mediated on-off mechanism of drug release (Fig. 6B). Improved release in lower $\mathrm{pH}$ compared to higher $\mathrm{pH}$ is attributed to the increased dissociation of electrostatically bound DOX molecules from PDA due to protonation of the $\mathrm{PDA}^{58}$. A clear acceleration of the drug release under NIR irradiation could be attributed to the local temperature increase due to the heating effect of PDA. Thus, increased temperature can affect the PCL layer to squeeze the drug molecules due to decreasing its drug holding capabilities by weakening the drug PCL interaction. The result clearly presents the NIR triggered drug delivery functionalities on an on-demand basis. Tuning of controlled drug release profile is a crucial aspect in the treatment of diseases. Precise and accurate delivery of the drug into the diseased site is important task in cancer treatment because it reduces the inevitable adverse effects of chemo which has a narrow therapeutic window.

In Vitro Photothermal Treatment. To evaluate the photothermal-chemo therapy effect, the different cells were incubated with PDP2 fibrous mats and subjected to $808 \mathrm{~nm}$ NIR laser irradiation as displayed in Fig. 7A. The CCK-8 assay was employed to determine the cell viability. As shown in Fig. 7B, PDP2 mat showed mild cytotoxicity $(70-80 \%)$ without NIR irradiation while same composite material showed the increased cytotoxicity on the applied NIR density-dependent manner. When applied NIR at more than $1.5 \mathrm{~W} / \mathrm{cm}^{2}$, cell viability was reduced significantly to cells that irradiated with $1 \mathrm{~W} / \mathrm{cm}^{2}$ or not irradiated mats (Fig. 7B).

Similarly, PDP0 exhibited a significant reduction of cell viability compared to not irradiated PDP2 mats or PDP2 mats with irradiation by $1 \mathrm{~W} / \mathrm{cm}^{2}$ (Fig. 7B). This result is accompanied by the burst release of DOX from PDP0 fiber into the cell medium, which directly induced cell deaths while PDA coated mats not subjected to NIR resulted in no apparent toxicity which is attributed to the coating of the biocompatible, non-toxic PDA over the drug-loaded mats. PDA coating prevents the DOX diffusion into medium somehow thereby lower toxicity in absence of external NIR. However, increased NIR exposure leads to significantly reduced cell viability compared to uncoated fiber is attributed to the synergistic effect of NIR itself which induced the hyperthermia, and NIR triggered increased drug release effect. NIR produces the local hyperthermia of $\sim 45$ and $>50^{\circ} \mathrm{C}$ for PDA coated mats irradiated with 1.5 and $2 \mathrm{~W} / \mathrm{cm}^{2}$, respectively. Tumor cells are highly vulnerable to the temperature higher than $42^{\circ} \mathrm{C}$, and cells undergo ablation at that temperature. The chemo-photothermal therapy effect of PDP2 was further investigated taking the PCL/PDA fiber without DOX (PP2). Results exhibited NIR irradiation exhibited remarkably reduced the cell viability for both cells type (Fig. 7B). This shows sufficient temperature induced by NIR itself can have cytotoxicity but notably, cell viability was observed significantly lower than the PDP2 in presence of NIR $\left(1.5 \mathrm{~W} / \mathrm{cm}^{2}\right)$. This result suggests the synergistic approach can lead to higher toxicity ${ }^{16,59}$. Furthermore, results of CCK assay was further counterchecked with the live and dead assay. Confocal imaging technique was used for the evaluation of live and dead MCF7 cells. Figure 8 shows the live cells are displayed in green while dead cells are in red. The microscopy observations revealed consistent with the result of CCK assay. Red cells are observed increasing with the increasing NIR laser power in NIR treated PDP2. The highest input of NIR laser resulted in the cells deaths almost completely. PDA has a high heating ability under the exposure to NIR. The mechanism of cell death via the synergistic effect of NIR mediated drug release and hyperthermia is proposed in schematically (Fig. 9).

The half maximum inhibitory concentration (IC50) value is the therapeutic concentration of drug which causes 50\% cancer cell mortality ${ }^{59,60}$. Table 3 shows the comparison of the IC50 values for different materials. As shown in Table 3, the IC50 value of free DOX was found $1.66 \pm 0.18 \mu \mathrm{m}$ which is lower compared to the values $1.98 \pm 0.13 \mu \mathrm{m}$ and $2.96 \pm 0.42 \mu \mathrm{m}$ obtained for PDP0 and PDP2, respectively. However, the application of the 


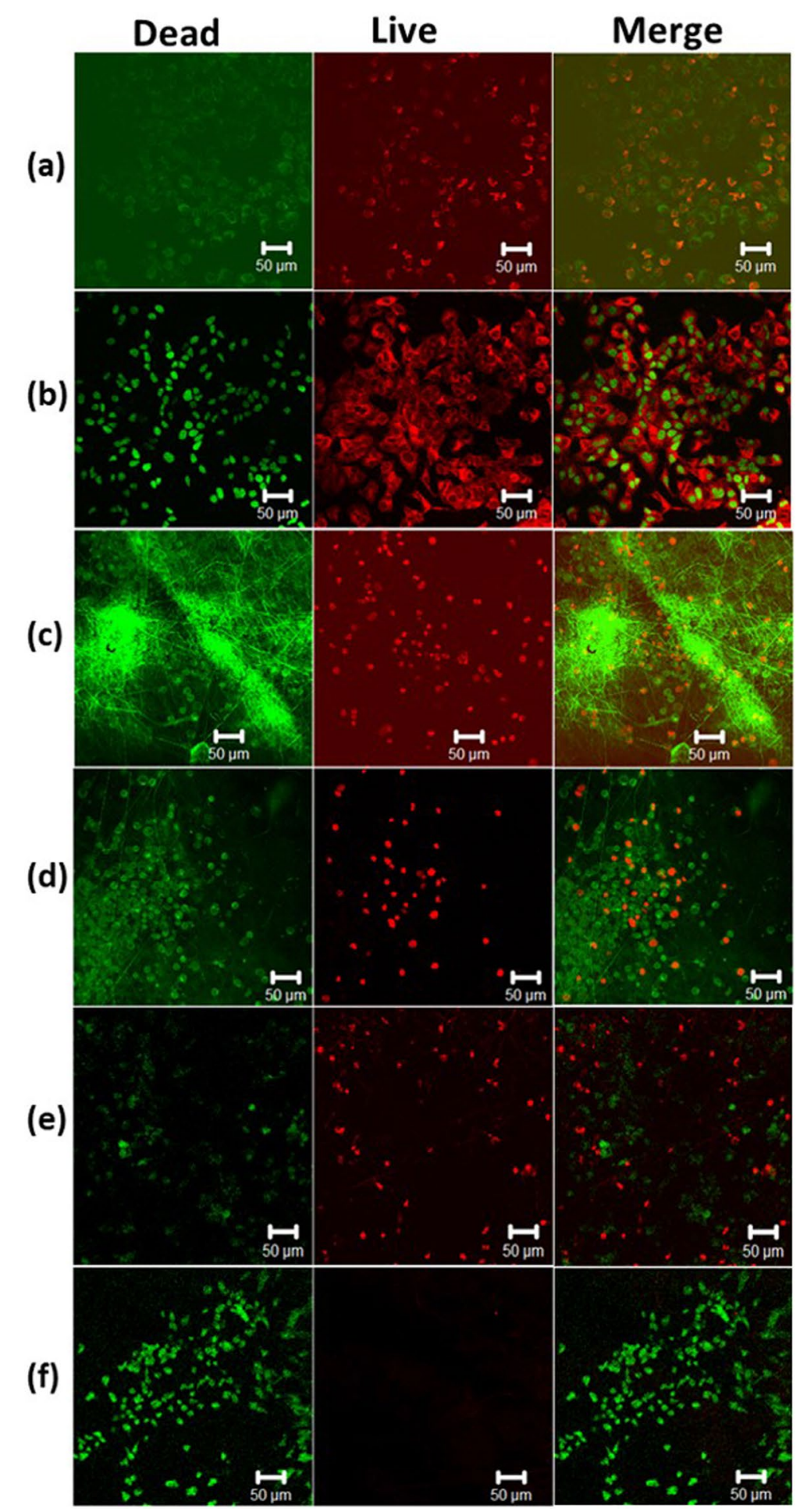

Figure 8. Confocal microscopy images of resazurin and SYTOX green stained MCF7 cells cultured on different membranes (a) PDP0, (b) PDP2 (without NIR), (c) PDP2 $\left(1 \mathrm{~W} / \mathrm{cm}^{2}\right)$, (d) PDP2 $\left(1.5 \mathrm{~W} / \mathrm{cm}^{2}\right),\left(\right.$ e) PP2 $\left(1.5 \mathrm{~W} / \mathrm{cm}^{2}\right)$ and (f) PDP2 $\left(2 \mathrm{~W} / \mathrm{cm}^{2}\right)$. Resazurin stains live cells red while SYTOX stains dead cells green.

NIR onto PDP2 mats showed the significantly lower IC50 values than those of PDP2. The reduction of the IC50 values was found dependently on irradiated NIR dose (Table 3). Induction of local hyperthermia due to NIR exposure of PDP2 mats and enhanced drug release due to hyperthermia synergistically could provide PDP2 mats with NIR irradiation with much lower IC50 value than the PDP2 mats not irradiated with NIR or PDP0 samples. In overall, in-vitro cells results indicated that PDA decoration into the drug-loaded fibers could be an effective localized platform that can inhibit tumor cell growth and eventual killing by delivering both a hyperthermia and heat mediated drug delivery under NIR exposure. 


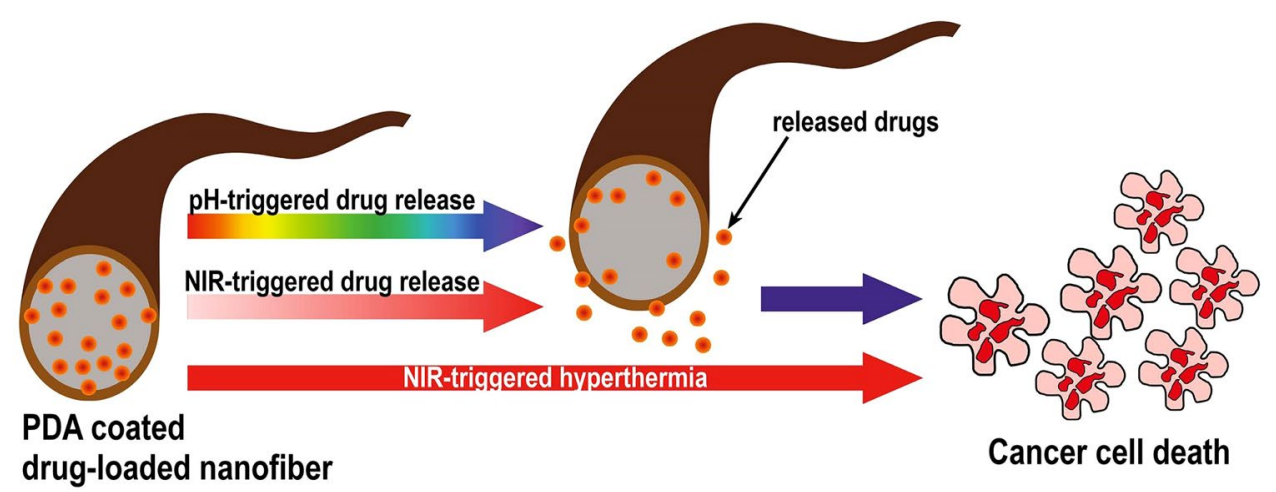

Figure 9. Schematic representation of cell death mechanism.

\begin{tabular}{|l|l|l|}
\hline Materials & NIR dose $\left(\mathbf{W} / \mathbf{c m}^{2}\right)$ & IC50 value $(\boldsymbol{\mu m})$ \\
\hline Free DOX & 0 & $1.66 \pm 0.18$ \\
\hline PDP0 & 0 & $1.98 \pm 0.13$ \\
\hline PDP2 & 0 & $2.96 \pm 0.42$ \\
\hline PDP2 & 1 & $1.61 \pm 0.10$ \\
\hline PDP2 & 2 & $0.43 \pm 0.04$ \\
\hline
\end{tabular}

Table 3. IC50 values of different samples.

\section{Conclusions}

We have reported the successful fabrication of localized anticancer drug delivery platform with bimodal functionalities. Facile electrospinning was used to fabricate PCL-DOX fiber and subsequent surface modification was performed by chemical polymerization of dopamine in various concentration. FESEM images confirmed the thin coating of the PCL-DOX membranes with extra nodules like PDA particles on the fiber surface. Resulting membranes exhibited the excellent photothermal behavior and stability in response to NIR laser of $808 \mathrm{~nm}$. Similarly, PDA coated mats have shown improved DOX release at $\mathrm{pH} 5.5$ than to higher $\mathrm{pH}$ solution (6.8 and 7.4). In addition, stimulation of PDA coated fibers by NIR light $\left(808 \mathrm{~nm}, 1.5 \mathrm{~W} / \mathrm{cm}^{2}, 5 \mathrm{~min}\right)$, the amount of DOX released in the local environment demonstrated the on-demand delivery of cancer drugs. Evaluation of the anticancer effect of PDA coated membranes showed superior toxicity in response to NIR illumination in compared to mats not irradiated, could be attributed to the synergistic effect of NIR driven photothermal therapy and improved DOX release simultaneously. This study provides a promising composite material, which can be used in the localized treatment of cancers and other diseases with tunable drug releasing properties.

\section{References}

1. Tang, R. et al. Direct Delivery of Functional Proteins and Enzymes to the Cytosol Using Nanoparticle-Stabilized Nanocapsules. ACS Nano 7, 6667-6673, https://doi.org/10.1021/nn402753y (2013).

2. Faraji, A. H. \& Wipf, P. Nanoparticles in cellular drug delivery. Bioorganic \& Medicinal Chemistry 17, 2950-2962, https://doi. org/10.1016/j.bmc.2009.02.043 (2009).

3. Zhang, J., Wang, X., Liu, T., Liu, S. \& Jing, X. Antitumor activity of electrospun polylactide nanofibers loaded with 5-fluorouracil and oxaliplatin against colorectal cancer. Drug Delivery 23, 784-790, https://doi.org/10.3109/10717544.2014.916768 (2016).

4. Sasikala, A. R. K., Unnithan, A. R., Yun, Y.-H., Park, C. H. \& Kim, C. S. An implantable smart magnetic nanofiber device for endoscopic hyperthermia treatment and tumor-triggered controlled drug release. Acta Biomaterialia 31, 122-133, https://doi. org/10.1016/j.actbio.2015.12.015 (2016).

5. Chen, Y. et al. DOX-Cu9S5@mSiO2-PG composite fibers for orthotopic synergistic chemo- and photothermal tumor therapy. Dalton Transactions 44, 3118-3127, https://doi.org/10.1039/C4DT03113J (2015).

6. Sasikala, A. R. K. et al. Multifaceted Implantable Anticancer Device for Potential Postsurgical Breast Cancer Treatment: A Single Platform for Synergistic Inhibition of Local Regional Breast Cancer Recurrence, Surveillance, and Healthy Breast Reconstruction. Advanced Functional Materials 28, 1704793, https://doi.org/10.1002/adfm.201704793 (2018).

7. Tiwari, A. P. et al. pH/NIR-Responsive Polypyrrole-Functionalized Fibrous Localized Drug-Delivery Platform for Synergistic Cancer Therapy. ACS Applied Materials \& Interfaces 10, 20256-20270, https://doi.org/10.1021/acsami.7b17664 (2018).

8. Aguilar, L. E. et al. Electrospun polyurethane/Eudragit ${ }^{\circledR}$ L100-55 composite mats for the $\mathrm{pH}$ dependent release of paclitaxel on duodenal stent cover application. International Journal of Pharmaceutics 478, 1-8, https://doi.org/10.1016/j.ijpharm.2014.10.057 (2015).

9. Irani, M., Mir Mohamad Sadeghi, G. \& Haririan, I. Electrospun biocompatible poly ( $\varepsilon$-caprolactonediol)-based polyurethane core/ shell nanofibrous scaffold for controlled release of temozolomide. International Journal of Polymeric Materials and Polymeric Biomaterials 67, 361-366, https://doi.org/10.1080/00914037.2017.1331350 (2018).

10. Ignatova, M, Rashkov, I. \& Manolova, N. Drug-loaded electrospun materials in wound-dressing applications and in local cancer treatment. Expert Opinion on Drug Delivery 10, 469-483, https://doi.org/10.1517/17425247.2013.758103 (2013).

11. Mahoney, B. P., Raghunand, N., Baggett, B. \& Gillies, R. J. Tumor acidity, ion trapping and chemotherapeutics: I. Acid pH affects the distribution of chemotherapeutic agents in vitro. Biochemical Pharmacology 66, 1207-1218, https://doi.org/10.1016/S00062952(03)00467-2 (2003). 
12. Fu, Y. et al. Constructing Implantable SrTiO3:Yb,Ho Nanofibers for NIR-Triggered and Optically Monitored Chemotherapy. Chemistry - A European Journal 23, 2423-2431, https://doi.org/10.1002/chem.201604956 (2017).

13. Jiang, J. et al. Mussel-inspired protein-mediated surface functionalization of electrospun nanofibers for $\mathrm{pH}$-responsive drug delivery. Acta Biomaterialia 10, 1324-1332, https://doi.org/10.1016/j.actbio.2013.11.012 (2014).

14. Chang, D. et al. Polydopamine-based surface modification of mesoporous silica nanoparticles as $\mathrm{pH}$-sensitive drug delivery vehicles for cancer therapy. Journal of Colloid and Interface Science 463, 279-287, https://doi.org/10.1016/j.jcis.2015.11.001 (2016).

15. Gao, N. et al. A Versatile Platform Based on Black Phosphorus Nanosheets with Enhanced Stability for Cancer Synergistic Therapy. 14, 1883-1897 (2018)

16. Liu, S. et al. Dynamically PEGylated and Borate-Coordination-Polymer-Coated Polydopamine Nanoparticles for Synergetic TumorTargeted. Chemo-Photothermal Combination Therapy. 14, 1703968, https://doi.org/10.1002/smll.201703968 (2018).

17. Tao, W. et al. Black Phosphorus Nanosheets as a Robust Delivery Platform for Cancer. Theranostics. 29, 1603276, https://doi. org/10.1002/adma.201603276 (2017).

18. Zeng, X. et al. Polydopamine-Modified Black Phosphorous Nanocapsule with Enhanced Stability and Photothermal Performance for Tumor Multimodal Treatments. Advanced Science 5, 1800510, https://doi.org/10.1002/advs.201800510 (2018).

19. Zhu, D. et al. Docetaxel (DTX)-loaded polydopamine-modified TPGS-PLA nanoparticles as a targeted drug delivery system for the treatment of liver cancer. Acta Biomaterialia 30, 144-154, https://doi.org/10.1016/j.actbio.2015.11.031 (2016).

20. Cheng, M. et al. Gold Nanorod-Embedded Electrospun Fibrous Membrane as a Photothermal Therapy Platform. ACS Applied Materials \& Interfaces 6, 1569-1575, https://doi.org/10.1021/am405839b (2014).

21. Peralta, D. V., Heidari, Z., Dash, S. \& Tarr, M. A. Hybrid Paclitaxel and Gold Nanorod-Loaded Human Serum Albumin Nanoparticles for Simultaneous Chemotherapeutic and Photothermal Therapy on 4T1 Breast Cancer Cells. ACS Applied Materials \& Interfaces 7, 7101-7111, https://doi.org/10.1021/acsami.5b00858 (2015).

22. Link, S., Burda, C., Mohamed, M. B., Nikoobakht, B. \& El-Sayed, M. A. Laser Photothermal Melting and Fragmentation of Gold Nanorods: Energy and Laser Pulse-Width Dependence. The Journal of Physical Chemistry A 103, 1165-1170, https://doi. org/10.1021/jp983141k (1999).

23. Han, X.-J. et al. Preparation and characterization of electrospun polyurethane/inorganic-particles nanofibers. Polymer Composites 33, 2045-2057, https://doi.org/10.1002/pc.22348 (2012).

24. Liu, Y., Ai, K. \& Lu, L. Polydopamine and Its Derivative Materials: Synthesis and Promising Applications in Energy, Environmental, and Biomedical Fields. Chemical Reviews 114, 5057-5115, https://doi.org/10.1021/cr400407a (2014)

25. Liu, Y. et al. Dopamine-Melanin Colloidal Nanospheres: An Efficient Near-Infrared Photothermal Therapeutic Agent for In Vivo Cancer Therapy. Advanced Materials 25, 1353-1359, https://doi.org/10.1002/adma.201204683 (2012).

26. GhavamiNejad, A., SamariKhalaj, M., Aguilar, L. E., Park, C. H. \& Kim, C. S. pH/NIR Light-Controlled Multidrug Release via a Mussel-Inspired Nanocomposite Hydrogel for Chemo-Photothermal Cancer Therapy. Scientific Reports 6, 33594, https://doi. org/10.1038/srep33594 (2016).

27. Cheng, W. et al. A Multifunctional Nanoplatform against Multidrug Resistant Cancer: Merging the Best of Targeted Chemo/Gene/ Photothermal Therapy. Advanced Functional Materials 27, 1704135, https://doi.org/10.1002/adfm.201704135 (2017).

28. Cheng, W. et al. TPGS-Functionalized Polydopamine-Modified Mesoporous Silica as Drug Nanocarriers for Enhanced Lung Cancer Chemotherapy against Multidrug Resistance. Small 13, 1700623, https://doi.org/10.1002/smll.201700623 (2017).

29. Liang, X. et al. Dye-conjugated single-walled carbon nanotubes induce photothermal therapy under the guidance of near-infrared imaging. Cancer Letters 383, 243-249, https://doi.org/10.1016/j.canlet.2016.09.006 (2016).

30. Fornari, F. et al. MiR-122/Cyclin G1 Interaction Modulates p53 Activity and Affects Doxorubicin Sensitivity of Human Hepatocarcinoma Cells. Cancer Research 69, 5761 (2009).

31. Tiwari, A. P. et al. Heterogeneous electrospun polycaprolactone/polyethylene glycol membranes with improved wettability, biocompatibility, and mineralization. Colloids and Surfaces A: Physicochemical and Engineering Aspects 520, 105-113, https://doi. org/10.1016/j.colsurfa.2017.01.054 (2017).

32. Li, Y. et al. Enhanced adhesion and proliferation of human umbilical vein endothelial cells on conductive PANI-PCL fiber scaffold by electrical stimulation. Materials Science and Engineering: C 72, 106-112, https://doi.org/10.1016/j.msec.2016.11.052 (2017).

33. He, M. et al. Fibrous guided tissue regeneration membrane loaded with anti-inflammatory agent prepared by coaxial electrospinning for the purpose of controlled release. Applied Surface Science 335, 121-129, https://doi.org/10.1016/j.apsusc.2015.02.037 (2015)

34. Bhattarai, D., Aguilar, L., Park, C. \& Kim, C. A Review on Properties of Natural and Synthetic Based Electrospun Fibrous Materials for Bone Tissue Engineering. Membranes 8, 62 (2018).

35. Wang, X. et al. A Polydopamine Nanoparticle-Knotted Poly(ethylene glycol) Hydrogel for On-Demand Drug Delivery and Chemophotothermal Therapy. Chemistry of Materials 29, 1370-1376, https://doi.org/10.1021/acs.chemmater.6b05192 (2017).

36. Cheng, W. et al. pH-Sensitive Delivery Vehicle Based on Folic Acid-Conjugated Polydopamine-Modified Mesoporous Silica Nanoparticles for Targeted Cancer Therapy. ACS Applied Materials \& Interfaces 9, 18462-18473, https://doi.org/10.1021/ acsami.7b02457 (2017).

37. Xu, X., Bai, B., Wang, H. \& Suo, Y. A Near-Infrared and Temperature-Responsive Pesticide Release Platform through Core-Shell Polydopamine@PNIPAm Nanocomposites. ACS Applied Materials \& Interfaces 9, 6424-6432, https://doi.org/10.1021/ acsami.6b15393 (2017).

38. Peng, Y. et al. A multifunctional nanoplatform for cancer chemo-photothermal synergistic therapy and overcoming multidrug resistance. Biomaterials Science 6, 1084-1098, https://doi.org/10.1039/C7BM01206C (2018).

39. Bhattarai, D. P. et al. Sacrificial template-based synthetic approach of polypyrrole hollow fibers for photothermal therapy. 534, 447-458 (2019).

40. Zeng, X. et al. Cholic acid-functionalized nanoparticles of star-shaped PLGA-vitamin E TPGS copolymer for docetaxel delivery to cervical cancer. Biomaterials 34, 6058-6067, https://doi.org/10.1016/j.biomaterials.2013.04.052 (2013).

41. Croisier, F. et al. Mechanical testing of electrospun PCL fibers. Acta Biomaterialia 8, 218-224, https://doi.org/10.1016/j. actbio.2011.08.015 (2012).

42. Tiwari, A. P., Joshi, M. K., Park, C. H. \& Kim, C. S. Nano-Nets Covered Composite Nanofibers with Enhanced Biocompatibility and Mechanical Properties for Bone Tissue Engineering. Journal of Nanoscience and Nanotechnology 18, 529-537, https://doi. org/10.1166/jnn.2018.13930 (2018).

43. Li, H., Zhu, J., Chen, S., Jia, L. \& Ma, Y. Fabrication of aqueous-based dual drug loaded silk fibroin electrospun nanofibers embedded with curcumin-loaded RSF nanospheres for drugs controlled release. RSC Advances 7, 56550-56558, https://doi.org/10.1039/ C7RA12394A (2017).

44. Zangmeister, R. A., Morris, T. A. \& Tarlov, M. J. Characterization of Polydopamine Thin Films Deposited at Short Times by Autoxidation of Dopamine. Langmuir 29, 8619-8628, https://doi.org/10.1021/la400587j (2013).

45. Elzubair, A., Elias, C. N., Suarez, J. C. M., Lopes, H. P. \& Vieira, M. V. B. The physical characterization of a thermoplastic polymer for endodontic obturation. Journal of Dentistry 34, 784-789, https://doi.org/10.1016/j.jdent.2006.03.002 (2006).

46. Xue, J. et al. Preparation and in vivo efficient anti-infection property of GTR/GBR implant made by metronidazole loaded electrospun polycaprolactone nanofiber membrane. International Journal of Pharmaceutics 475, 566-577, https://doi.org/10.1016/j. ijpharm.2014.09.026 (2014)

47. Tiwari, A. P. et al. Formation of lipophilic drug-loaded human serum albumin nanofibers with the aid of glutathione. Chemical Engineering Journal 313, 753-758, https://doi.org/10.1016/j.cej.2016.12.121 (2017). 
48. Zeng, X. et al. A Drug-Self-Gated Mesoporous Antitumor Nanoplatform Based on pH-Sensitive Dynamic Covalent Bond. Advanced Functional Materials 27, 1605985, https://doi.org/10.1002/adfm.201605985 (2017).

49. Nie, J. et al. Co-delivery of docetaxel and bortezomib based on a targeting nanoplatform for enhancing cancer chemotherapy effects. 24, 1124-1138 (2017)

50. Mičušík, M., Omastová, M., Prokeš, J. \& Krupa, I. Mechanical and electrical properties of composites based on thermoplastic matrices and conductive cellulose fibers. Journal of Applied Polymer Science 101, 133-142, https://doi.org/10.1002/app.23041 (2006).

51. Stella, J. A., D’Amore, A., Wagner, W. R. \& Sacks, M. S. On The Biomechanical Function of Scaffolds For Engineering Load Bearing Soft Tissues. Acta biomaterialia 6, 2365-2381, https://doi.org/10.1016/j.actbio.2010.01.001 (2010).

52. Zeng, J. et al. Influence of the drug compatibility with polymer solution on the release kinetics of electrospun fiber formulation. Journal of Controlled Release 105, 43-51, https://doi.org/10.1016/j.jconrel.2005.02.024 (2005).

53. Zhou, M. et al. Interfacial crystallization enhanced interfacial interaction of Poly (butylene succinate)/ramie fiber biocomposites using dopamine as a modifier. Composites Science and Technology 91, 22-29, https://doi.org/10.1016/j.compscitech.2013.11.019 (2014).

54. Chen, S., Cao, Y. \& Feng, J. Polydopamine As an Efficient and Robust Platform to Functionalize Carbon Fiber for High-Performance Polymer Composites. ACS Applied Materials \& Interfaces 6, 349-356, https://doi.org/10.1021/am404394g (2014).

55. He, X., Wolkers, W. F., Crowe, J. H., Swanlund, D. J. \& Bischof, J. C. In Situ Thermal Denaturation of Proteins in Dunning AT-1 Prostate Cancer Cells: Implication for Hyperthermic Cell Injury. Annals of Biomedical Engineering 32, 1384-1398, https://doi. org/10.1114/B:ABME.0000042226.97347.de (2004).

56. Tran, T. H. et al. Development of a Graphene Oxide Nanocarrier for Dual-Drug Chemo-phototherapy to Overcome Drug Resistance in Cancer. ACS Applied Materials \& Interfaces 7, 28647-28655, https://doi.org/10.1021/acsami.5b10426 (2015).

57. Wu, S. et al. Adsorption Properties of Doxorubicin Hydrochloride onto Graphene Oxide: Equilibrium, Kinetic and Thermodynamic Studies. Materials 6, 2026-2042, https://doi.org/10.3390/ma6052026 (2013).

58. Das, D. et al. Biocompatible nanogel derived from functionalized dextrin for targeted delivery of doxorubicin hydrochloride to MG 63 cancer cells. Carbohydrate Polymers 171, 27-38, https://doi.org/10.1016/j.carbpol.2017.04.068 (2017).

59. Li, D. et al. Biomimetic albumin-modified gold nanorods for photothermo-chemotherapy and macrophage polarization modulation. Acta Pharmaceutica Sinica B 8, 74-84, https://doi.org/10.1016/j.apsb.2017.09.005 (2018).

60. Liang, C. et al. Self-controlled release of Oxaliplatin prodrug from d- $\alpha$-tocopheryl polyethylene glycol 1000 succinate (TPGS) functionalized mesoporous silica nanoparticles for cancer therapy. Journal of Colloid and Interface Science 525, 1-10, https://doi. org/10.1016/j.jcis.2018.04.058 (2018).

\section{Acknowledgements}

This paper was supported by a grant from the Basic Science and Technology (Project No. 2016R1A2A2A07005160). And also partially supported by the program for fostering next-generation researchers in engineering of National Research Foundation of Korea (NRF) funded by the Ministry of Science (Project No. 2017H1D8A2030449).

\section{Author Contributions}

A.P.T., H.Y.K., C.H.P. and C.S.K. designed the work; A.P.T., B.M., D.P.B., and S.W.K. carried out the research, prepared the data, and analyzed the data; A.P.T., H.Y.K., C.H.P. and C.S.K. wrote the main manuscript text and supervised this project. All authors reviewed the manuscript.

\section{Additional Information}

Competing Interests: The authors declare no competing interests.

Publisher's note: Springer Nature remains neutral with regard to jurisdictional claims in published maps and institutional affiliations.

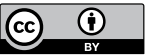

Open Access This article is licensed under a Creative Commons Attribution 4.0 International License, which permits use, sharing, adaptation, distribution and reproduction in any medium or format, as long as you give appropriate credit to the original author(s) and the source, provide a link to the Creative Commons license, and indicate if changes were made. The images or other third party material in this article are included in the article's Creative Commons license, unless indicated otherwise in a credit line to the material. If material is not included in the article's Creative Commons license and your intended use is not permitted by statutory regulation or exceeds the permitted use, you will need to obtain permission directly from the copyright holder. To view a copy of this license, visit http://creativecommons.org/licenses/by/4.0/.

(C) The Author(s) 2019 